ARTICLE OPEN

\title{
Suppression of superconductivity and enhanced critical field anisotropy in thin flakes of FeSe
}

\author{
Liam S. Farrar $\mathbb{D}^{1 凶}$, Matthew Bristow $\mathbb{D}^{2}$, Amir A. Haghighirad ${ }^{2,3}$, Alix McCollam ${ }^{4}$, Simon J. Bending ${ }^{1}$ and Amalia I. Coldea $\mathbb{D}^{2 凶}$
}

FeSe is a unique superconductor that can be manipulated to enhance its superconductivity using different routes, while its monolayer form grown on different substrates reaches a record high temperature for a two-dimensional system. In order to understand the role played by the substrate and the reduced dimensionality on superconductivity, we examine the superconducting properties of exfoliated FeSe thin flakes by reducing the thickness from bulk down towards $9 \mathrm{~nm}$. Magnetotransport measurements performed in magnetic fields up to $16 \mathrm{~T}$ and temperatures down to $2 \mathrm{~K}$ help to build up complete superconducting phase diagrams of different thickness flakes. While the thick flakes resemble the bulk behaviour, by reducing the thickness the superconductivity of FeSe flakes is suppressed. The observation of the vortex-antivortex unbinding transition in different flakes provide a direct signature of a dominant two-dimensional pairing channel. However, the upper critical field reflects the evolution of the multi-band nature of superconductivity in FeSe becoming highly two-dimensional and strongly anisotropic only in the thin limit. Our study provides detailed insights into the evolution of the superconducting properties of a multi-band superconductor FeSe in the thin limit in the absence of a dopant substrate.

npj Quantum Materials (2020)5:29; https://doi.org/10.1038/s41535-020-0227-3

\section{INTRODUCTION}

Amongst iron-based superconductors, FeSe has the simplest stoichiometric crystal structure, making it an ideal candidate to study the mechanisms of superconductivity ${ }^{1}$. Two-dimensional FeSe has attracted much interest due to the discovery of high temperature superconductivity in monolayer FeSe grown on $\mathrm{SrTiO}_{3}^{2-5}$, which is one of the highest critical temperatures amongst all iron-based superconductors. In addition, due to the weak van der Waals bonding of the FeSe layers, the material cleaves readily and has potential applications in heterostructure devices ${ }^{6,7}$. It is therefore important to understand any changes in the properties of the material as it is thinned towards the monolayer limit.

Previous studies examining the thickness dependence of FeSe have been limited to measurements on thin films grown using techniques such as molecular beam expitaxy ${ }^{8-10}$, pulsed laser deposition $^{11}$ and DC sputtering ${ }^{12}$, all of which require welloptimised growth protocols. The resulting thin films are strongly susceptible to interaction with the growth substrate, due to factors such as strain and charge transfer ${ }^{5}$. As the thickness of the FeSe films is reduced, superconductivity is usually suppressed, resulting in a superconductor-insulator transition either caused by disorder or strain induced by the change in the lattice parameters ${ }^{12,13}$. In $\mathrm{FeSe} / \mathrm{SrTiO}_{3}$, superconductivity in thin films can be enhanced by high temperature annealing that leads to changes in the carrier doping at the interface 9 . It is therefore important to establish the necessary ingredients to enhance superconductivity in FeSe in the absence of a strong substrate interaction that can induce direct doping, as well as applies strain.

An alternative to the growth of thin films is to create devices by mechanical exfoliation of high quality single crystals. This has proven extremely successful in the case of the layered superconductors $\mathrm{NbSe}_{2}{ }^{14}, \mathrm{TaS}_{2}{ }^{15}$ and $\mathrm{Bi}_{2} \mathrm{Sr}_{2} \mathrm{CaCu}_{2} \mathrm{O}_{8+\delta}{ }^{16}$, in which the inherent thickness-dependence of the superconducting transition has has been measured down to a monolayer. Recently, exfoliated FeSe devices have been realised ${ }^{17-19}$, with samples displaying superconducting behaviour at a thickness where thin films of FeSe are typically insulating ${ }^{12}$. However, these samples exhibit a suppressed superconducting critical temperature when compared to the bulk crystals from which they were exfoliated. One possible factor in suppressing superconductivity is the sample degradation caused by multiple fabrication steps, as well as long term exposure to air $^{20}$. It is therefore important that any study of the thickness dependence of superconductivity of FeSe utilise a fabrication method free from the harsh chemicals and high temperatures involved in traditional lithographic processing.

In this work we present a detailed study of the nature of superconductivity in ultra-thin flakes of FeSe fabricated utilising a deterministic transfer method ${ }^{21}$. We use magnetotransport measurements in high magnetic fields up to $16 \mathrm{~T}$ to investigate the effect of thickness on this material's superconducting properties. As the thickness is reduced from $100 \mathrm{~nm}$ towards $9 \mathrm{~nm}$, we detect that the upper critical field shows a crossover towards a two-dimensional character in superconductivity that manifests as a significant enhancement in the anisotropy of the upper critical field. Furthermore, the observation of the Berezinskii-Kosterlitz-Thouless transition provides important insights into the dominant intrinsic two-dimensional nature of multi-band superconductivity of FeSe, in the absence of the strong interaction with substrate or doping effects.

\section{RESULTS}

Thickness dependence of the transport behaviour of FeSe thin flakes

Figure 1a shows the typical temperature-dependence of the normalised resistance $(R(\mathrm{~T}) / R(300 \mathrm{~K}))$ for a bulk crystal and five thin

\footnotetext{
${ }^{1}$ Centre for Nanoscience and Nanotechnology, University of Bath, Bath BA2 7AY, UK. ${ }^{2}$ Clarendon Laboratory, University of Oxford, Parks Road, Oxford OX1 3PU, UK. ${ }^{3}$ Institute for Quantum Materials and Technologies (IQMT), Karlsruhe Institute of Technology, 76021 Karlsruhe, Germany. ${ }^{4}$ High Field Magnet Laboratory (HFML-EMFL), Radboud University, Toernooiveld 7, 6525 ED Nijmegen, The Netherlands. ${ }^{凶}$ email: L.S.Farrar@bath.ac.uk; amalia.coldea@physics.ox.ac.uk
} 

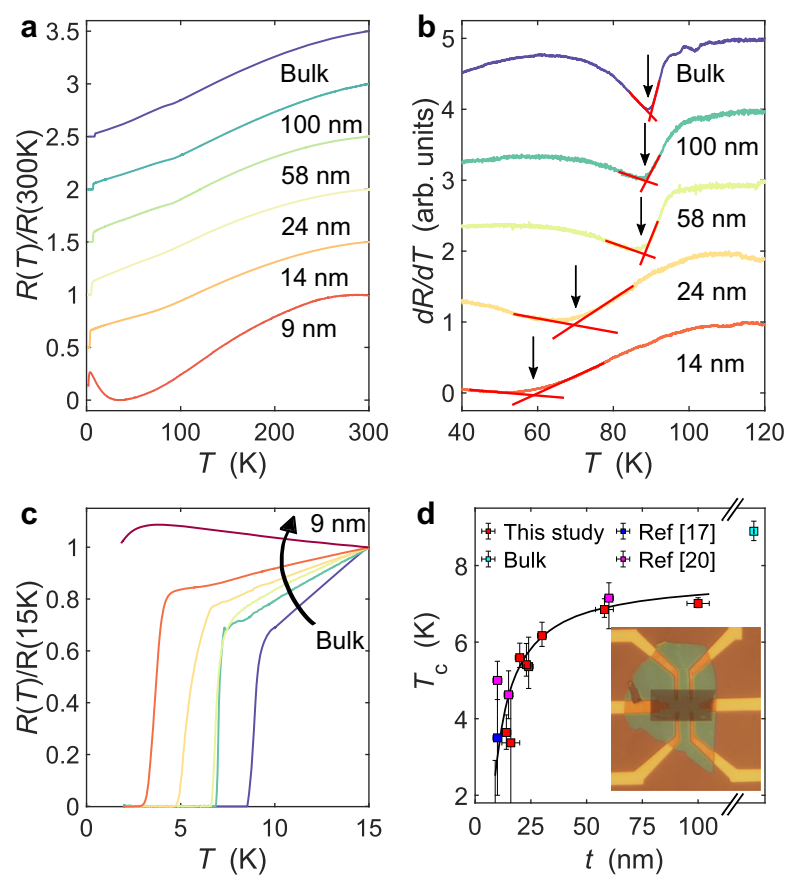

Fig. 1 Thickness dependence of the transport properties of FeSe thin flakes. a Temperature dependence of the normalised resistance $R(T) / R(300 \mathrm{~K})$ for a bulk crystal and five different thin flakes $(t=9-$ $100 \mathrm{~nm}$ ). $\mathbf{b}$ The high-temperature $T$-dependence of the normalised derivative of resistance, $\mathrm{d} R / \mathrm{d} T$, for the bulk sample and four thin flakes in a. The arrows indicate the position of the structural transition at $T_{\mathrm{s}}$. The curves in $\mathbf{a}$ and $\mathbf{b}$ are shifted vertically for clarity. c The low temperature superconducting transitions as in a but scaled to $R(T) / R(15 \mathrm{~K})$. d Thickness dependence of the superconducting critical temperature for several thin flake samples measured in this study, along with data from refs. ${ }^{17,20}$. The solid line is a fit to the Cooper model ${ }^{25,26}$. The inset shows an optical image of a $14 \mathrm{~nm}$ FeSe device capped with a thin layer $(\sim 20 \mathrm{~nm})$ of h-BN. The scale bar corresponds to $10 \mu \mathrm{m}$. See Methods for definition of error bars.

flake devices with thickness $t$ in the range $9-100 \mathrm{~nm}$ (see the Supplementary Material for additional devices). We observe significant changes in the transport behaviour of our devices which are highly dependent on the flake thickness. Firstly, the thick flake devices with $t \geq 58 \mathrm{~nm}$ are of highly quality with large residual resistance ratio (RRR) (see Fig. 1). They display similar transport behaviour to bulk $\mathrm{FeSe}^{22}$, in which the nematic phase transition occurs around $T_{\mathrm{s}} \sim 89 \mathrm{~K}$ accompanied by a superconducting transition at $T_{\mathrm{c}} \sim 9 \mathrm{~K}$ (see Fig. 1b). We notice that $T_{\mathrm{c}}$ of all thick flake devices is slightly lower than in bulk, with a maximum of $T_{\mathrm{c}} \sim 7 \mathrm{~K}$ for the $100 \mathrm{~nm}$ flake despite a relatively high RRR of 16 and a sharp transition width, $\Delta T_{c}$ of $0.3 \mathrm{~K}$. Next, in thinner flake devices we observe a systematic suppression of superconductivity, accompanied by a broadening of the resistive transition width, as shown in Fig. 1c). Lastly, the thinnest device reported here with $t=9 \mathrm{~nm}$ displays an anomalous upturn in resistance at low temperature, before a sharp decrease near $3 \mathrm{~K}$, indicating that a superconducting phase may only be stabilised below the experimental temperature limit of $2 \mathrm{~K}$.

Another important signature in the transport data of FeSe is the emergence of the nematic phase that triggers a tetragonal to orthorhombic structural transition at $T_{\mathrm{s}}$ and causes significant inplane distortion of the Fermi surface ${ }^{22}$. Fig. $1 \mathrm{~b}$ shows that the nematic transition has a sharp anomaly identified by the minimum in $\mathrm{d} R / \mathrm{d} T$ that is slightly suppressed for thick flakes $(t>50 \mathrm{~nm})$, as compared with bulk single crystals of FeSe. However, in thinner samples $(t<50 \mathrm{~nm})$ this transition is ill-defined and appears to be significantly reduced, as shown in Supplementary Fig. S1b and Fig. S3. This behaviour is reminiscent of that found in polycrystalline samples of $\mathrm{FeSe}^{1}$, or $\mathrm{Cu}$-doped $\mathrm{FeSe}^{23}$, in which the RRR is reduced as the degree of disorder and local inhomogeneity is much higher (see Supplementary Fig. S1), in comparison to high quality single crystals of FeSe in which quantum oscillations have been observed $^{24}$.

A summary showing how the superconductivity of thin flakes of FeSe is affected by the thickness reduction is shown in Fig. 1d. While $T_{\mathrm{c}}$ remains relatively constant for thicker flakes $(50-100 \mathrm{~nm})$, a sharp decrease in superconductivity occurs for the thinnest flakes $(t<25 \mathrm{~nm})$. We can describe the observed superconducting behaviour using the Cooper-law given by $T_{c} \sim \exp \left(-t_{m} / t\right)$, where $t_{m}=2 a /\left(N_{0} V\right), a$ is the Thomas Fermi screening length and $N_{0} V$ is the bulk pairing potential ${ }^{25,26}$. Since $a$ is inversely proportional to the square root of the density of states at the Fermi energy, this behaviour is expected for systems with very small Fermi energy, as found in $\mathrm{FeSe}^{22}$. The Cooper-law is commonly used to describe superconducting thin films but the trends observed in our data are in qualitative agreement with those found in thin films of $\mathrm{FeSe}^{12}$, thin flakes of $\mathrm{FeSe}_{0.3} \mathrm{Te}_{0.7}{ }^{27}$ and nanoflakes devices of FeSe fabricated using alternate device fabrication techniques ${ }^{17,20}$.

\section{BKT transition in FeSe thin flakes}

Next, we focus on other manifestations of superconductivity in thin flake devices of FeSe. The appearance of a BerezinskiiKosterlitz-Thouless (BKT) transition in a material is a signature of a $2 \mathrm{D}$ superconducting state ${ }^{28,29}$. This arises due to the thermal nucleation of vortex-antivortex pairs in the absence of an external magnetic field. Vortex-antivortex unbinding gives rise to dissipation, which results in a resistive transition even when the temperature is below the mean field pairing temperature. Just above the critical current, $I_{\mathrm{c}}$, the $I V$ curves follow the $V \propto I^{a(T)}$ dependence, where $a$ is a temperature-dependent exponent. At $T_{\text {BKT }}$ the critical exponent, $a$, abruptly increases from 1 at higher temperatures, due to flux flow of thermally dissociated vortexantivortex pairs to 3 at lower temperatures due to the currentdriven dissociation of vortex-antivortex pairs ${ }^{30,31}$.

In order to determine whether BKT physics plays an important role in the observed suppression of superconductivity in FeSe thin flakes, we investigate the temperature-dependent current-voltage characteristics for two devices with $t=14 \mathrm{~nm}$ and $t=100 \mathrm{~nm}$ at temperatures near $T_{c}$, as shown in Fig. 2a, b. We find a non-linear behaviour in the high current regime suggesting a currentinduced vortex-antivortex depairing, as expected for a BKT transition, without displaying the sudden jump in $a(T)$. The value of the exponent reaches the critical value of $a=3$ at $T_{\mathrm{BKT}}=2.9 \mathrm{~K}$ for the $t=14 \mathrm{~nm}$ sample and $T_{\mathrm{BKT}}=6.67 \mathrm{~K}$ for the $t=100 \mathrm{~nm}$ sample, as shown in Fig. $2 \mathrm{C}$, d. In both cases, the calculated $T_{\mathrm{BKT}}$ lies below the temperature (see also Supplementary Fig. S9) at which the resistance is $1 \%$ of the normal state value, suggesting that BKT physics is not the cause of the suppression of superconductivity in thin flakes. This signature of the BKT transition in FeSe flakes of different thickness is in qualitative agreement with previous reports on monolayer $\mathrm{FeSe} / \mathrm{SrTiO}_{3}$ and $500 \mathrm{~nm}$ thick films of $\mathrm{FeSe}^{32,33}$. Furthermore, signatures of dominant two-dimensional superconducting fluctuations have been observed in single crystals of LiFeAs ${ }^{34}$. The BKT behaviour suggests that the superconductivity of FeSe is quasi-two dimensional and this can be a general signature of multi-band ironbased superconductors where 2D fluctuations can be responsible for the suppression of superconductivity. The lack of the sudden jump in $a$ at $T_{\text {BKT }}$ and the non-linear $I V$ behaviour has been found in other thin films of conventional superconductors, where the disorder smears out the sharp features ${ }^{35}$. In our FeSe devices local inhomogeneities caused by the formation of structural domains at temperatures below the nematic transition can lead to an increase 

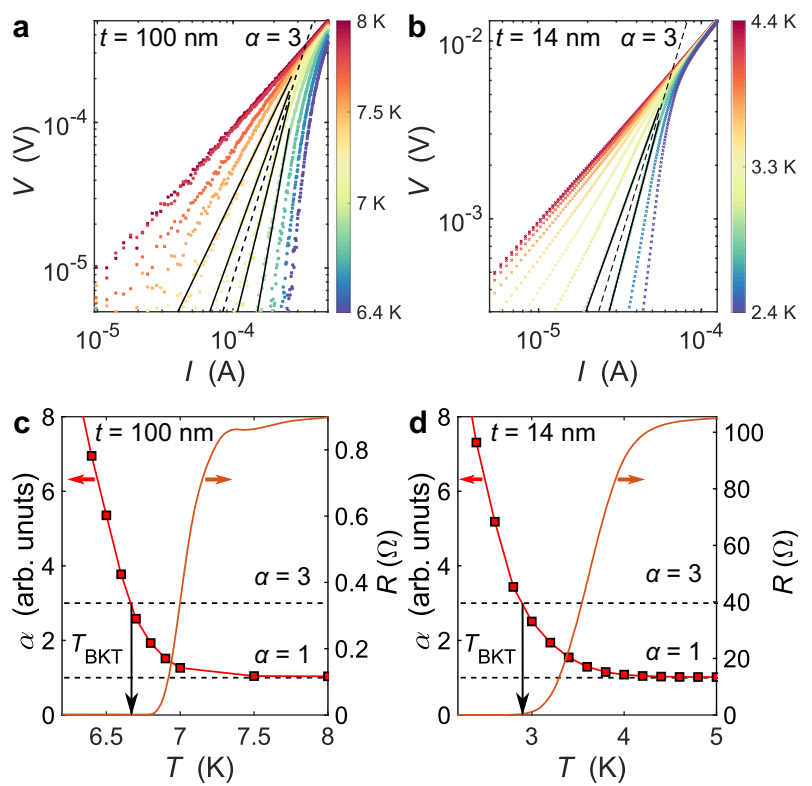

Fig. 2 BKT behaviour in FeSe thin flakes. a, b Voltage-current (IV) curves for $100 \mathrm{~nm}$ and $14 \mathrm{~nm}$ FeSe devices at different temperatures in the vicinity of $T_{\mathrm{c}}$. The solid black lines are linear fits to the low current regime used to extract the exponent $a$ from the $V \propto l^{\alpha(T)}$ dependence. $\mathbf{c}, \mathbf{d}$ Plot of the exponent $a$ extracted from $\mathbf{a}, \mathbf{b}$ ) (left axis) and the resistance transition (right axis) as a function of temperature. $a(T)$ reaches a value of 3 at $T_{\mathrm{BKT}}=6.67 \mathrm{~K}$ for the $100 \mathrm{~nm}$ and $T_{\mathrm{BKT}}=2.9 \mathrm{~K}$ for $14 \mathrm{~nm}$ device. The location of $T_{\mathrm{BKT}}$ is indicated by an arrow.

in the width of the superconducting transition. However, for our thinnest device of $14 \mathrm{~nm}$ (made out of 25 monolayers of FeSe) the sheet resistance is $\sim 233 \Omega / \square$ (where $\Omega / \square$ is the standard unit of sheet resistance) which is still much lower than the quantum resistance required to cause localisation (the mean free path reaches $\ell \sim 6.5 \mathrm{~nm}$ for $14 \mathrm{~nm}$ flake $)^{36}$.

Enhancement of the upper critical field anisotropy in FeSe thin flakes

A key feature of two-dimensional superconductivity is the significant enhancement of anisotropy in the angular dependence of the upper critical field $H_{\mathrm{c} 2}{ }^{14}$. To investigate the suppression of superconducting behaviour in a magnetic field we have measured the resistance of several devices for different orientations of applied field. Figures $3 a$, b show the temperature dependence of the resistive transition of a thin flake device with $t=14 \mathrm{~nm}$ in constant magnetic fields up to $0.8 \mathrm{~T}$ with $H \| c$, and in fields up to $8 \mathrm{~T}$ with $H \|(a b)$. Additional transport measurements as a function of magnetic field performed at different fixed temperatures, as well as at fixed angles $\theta$ are shown in Supplementary Fig. S6. Based on these measurements, one can construct the phase diagram of the upper critical field for several different devices, as summarised in Fig. 3d.

In addition, to assess the changes in the superconducting anisotropy at the lowest experimental temperature we have performed an angle-dependent study of the upper critical field $H_{\mathrm{c} 2}(\theta)$ for two devices with $t=14 \mathrm{~nm}$ and $t=24 \mathrm{~nm}$ at $T=2 \mathrm{~K}$, as presented in Fig. $3 c$ using a methodology presented in Fig. 8. To be able to compare the two different devices, we plot the ratio $\left.H_{\mathrm{c} 2}(\theta) / H_{\mathrm{c} 2}(\theta=0)\right)$, as shown in Fig. 4c. Using the anisotropic Ginzburg-Landau (GL) theory ${ }^{37}$, we can extract the anisotropy parameter $\Gamma$ defined by the ratio between $H_{\mathrm{c} 2}$ when $H \|(a b)$ to $H_{\mathrm{c} 2}$ for $H \| c$. We find that $\Gamma$ increases significantly from 2.4 for the $t=$ $24 \mathrm{~nm}$ device to $>10$ when $t=14 \mathrm{~nm}$. This indicates a significant
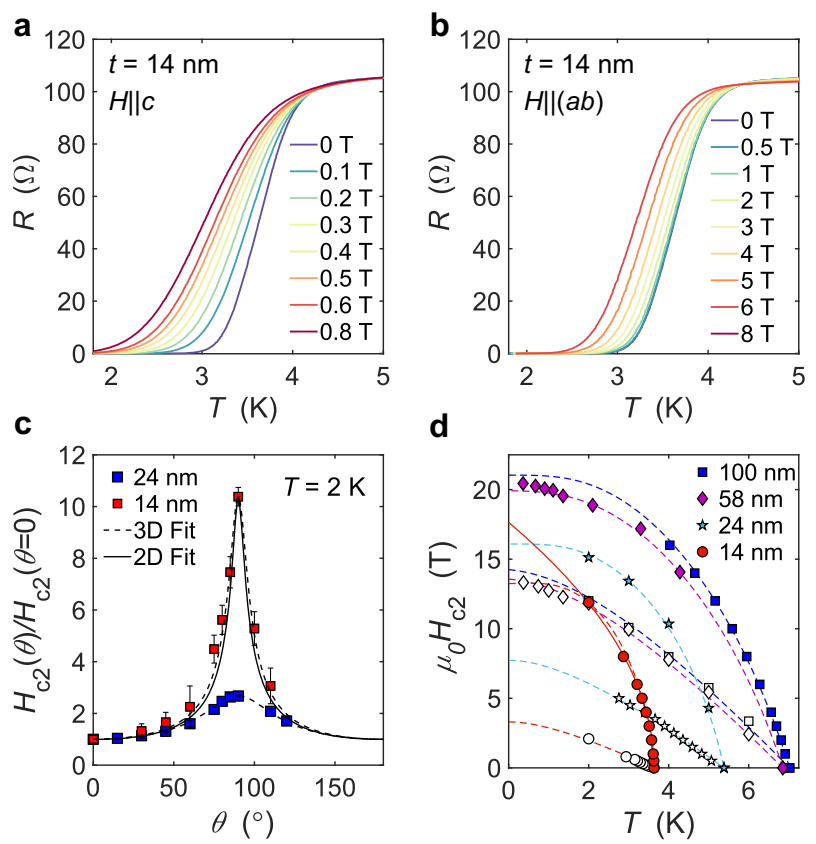

Fig. 3 Thickness dependence of the upper critical field anisotropy $H_{\mathrm{c}_{2}}(\boldsymbol{\theta})$ of FeSe thin flakes. $\mathbf{a}$, b Superconducting transition of a $t=$ $14 \mathrm{~nm}$ FeSe thin flake device as a function of magnetic field applied parallel and perpendicular to the crystallographic $c$-axis, respectively. c Angular dependence of the superconducting upper critical field, $H_{\mathrm{c} 2}(\theta)$, at $2 \mathrm{~K}$ for devices with thickness of $t=14 \mathrm{~nm}$ and $t=$ $24 \mathrm{~nm}$ (raw data are shown in Supplementary Fig. S6) Dashed line represents a fit to the Ginzburg-Landau model for a type-II anisotropic superconductor and the solid line represents a fit to the 2D-GL model ${ }^{42}$. Here $H_{\mathrm{c} 2}$ is defined as the offset of superconductivity rather than the resistive transition midpoint as in the other cases. See Methods for definition of error bars. d The temperature dependence of the upper critical field for four FeSe devices with the magnetic field applied either parallel (open symbols) or perpendicular (closed symbols) to the crystallographic $c$-axis. Dashed lines represent fits to the $\mathrm{WHH}$ orbital pair-breaking model and the solid line represents a fit to the 2D-GL expression for $H_{\mathrm{C} 2}(T)^{37}$.

increase in anisotropy as the flakes become thinner and closer to the two-dimensional limit, as shown in Fig. 4b (additional data for a $t=16 \mathrm{~nm}$ flake is shown in Fig. 7). The value for the thicker flake device is comparable to the value of 1.8 observed in bulk FeSe crystals $^{38}$, while the thinner flake device has a large anisotropy, comparable to that observed in FeS crystals ${ }^{39}$. This suggests that the enhanced anisotropy can be linked to an increase in twodimensionality of the Fermi surface.

As the superconducting anisotropy, $\Gamma$, is strongly temperature dependent, we analyse in detail the complete superconducting phase diagram as a function of magnetic fields parallel and perpendicular to the conducting planes for different devices, as shown in Fig. 3d. We find that the standard three-dimensional Werthamer-Helfand-Hohenberg $(\mathrm{WHH})$ model $^{40}$, with the inclusion of spin paramagnetism and spin-orbit scattering, describes the temperature dependence of the upper critical field of the thick $100 \mathrm{~nm}$ flake device. A list of all obtained parameters can be found in Supplementary Table S1. Orbital pair breaking alone accounts for the temperature dependence of $H_{c 2}$ for $H \| c$, as shown in Fig. $3 d$. However, when the magnetic field is aligned along the conducting $(a b)$ plane, a Pauli pair breaking contribution has to be included which reduces the orbital-limited critical field by $\mu_{0} H_{\mathrm{c} 2}^{\text {orb }} / \sqrt{1+a_{M}^{2}}$, where $a_{\mathrm{M}}$ is the Maki parameter. The extracted 

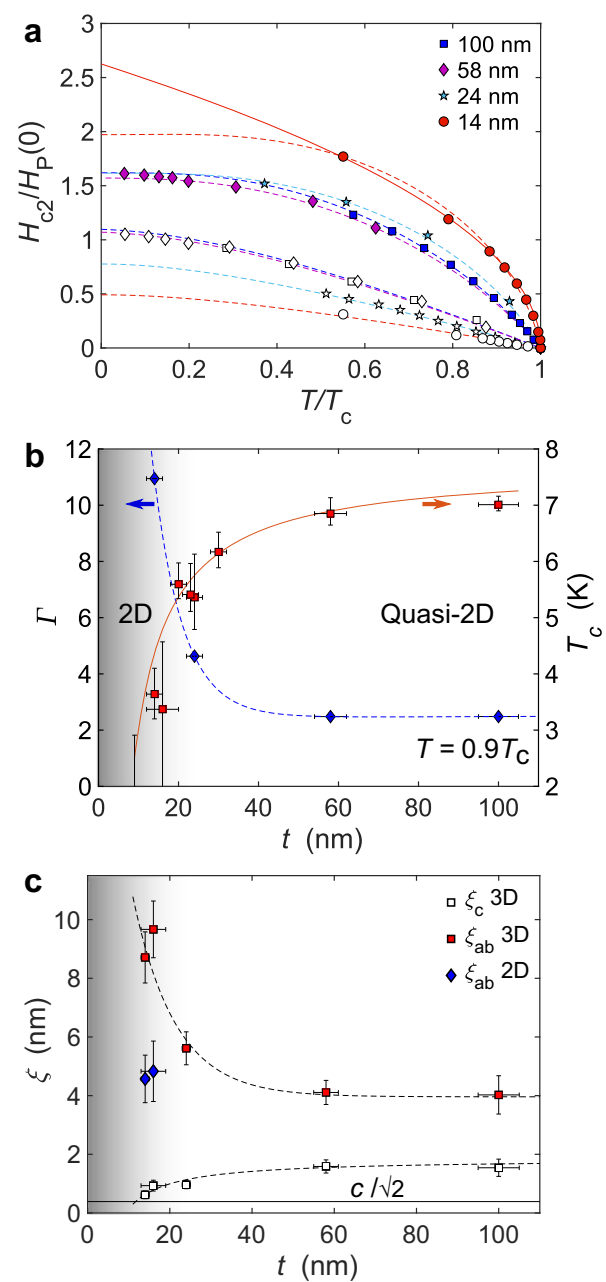

Fig. 4 Thickness dependence of the reduced upper critical field $H_{\mathrm{c} 2} / H_{P}(\theta)$, critical temperature $T_{c}$, anisotropy $\Gamma$, and coherence lengths $\boldsymbol{\xi}_{\mathrm{ab}}, \boldsymbol{\xi}_{\mathrm{c}}$ of FeSe thin flakes. a The reduced superconducting critical field $H_{c 2} / H_{p}(0)$ as a function of reduced transition temperature $T / T_{\mathrm{c}}$ for magnetic field applied either parallel (open symbols) or perpendicular (closed symbols) to the crystallographic c-axis for devices of different thickness. Dashed lines represent fits to the WHH orbital pair-breaking model and the solid line represents a fit to a 2D-Ginzburg Landau model ${ }^{42}$. b The anisotropy parameter, $\Gamma$, defined by the ratio of $H_{c 2}$ for the two field orientations presented in a at $0.9 T_{\mathrm{c}}$ (left axis) and $T_{\mathrm{c}}$ (right axis) as a function of thickness, $t$. c The coherence lengths obtained from the slope of $H_{C 2}$ in the vicinity of $T_{c}$ as detailed in the Supplementary Material. The horizontal solid line indicates the $c / \sqrt{2}$-axis layer spacing of bulk FeSe. The shaded area indicates the crossover towards a two-dimensional highly anisotropic superconducting phase, and the dashed lines in $\mathbf{b}$ and $\mathbf{c}$ are guides to the eyes. See Methods for definition of error bars.

Maki parameter $a_{\mathrm{M}}$ is 2.4 for thick flakes ( $t=54$ and $100 \mathrm{~nm}$ ), close to the value of $a_{M}=2.1$ found for bulk single crystals ${ }^{38}$. For a thinner flake $(t=24 \mathrm{~nm})$, the Maki parameter increases to 4.15 . To describe the data fully the spin-orbit scattering constant needs to be included and varies from $\lambda_{\text {so }} \sim 0.2-0.35$ (see Supplementary Fig. S5). Our WHH fitting parameters are close to the values obtained for $\mathrm{FeSe}_{0.6} \mathrm{Te}_{0.6}$ single crystals where $a_{\mathrm{M}} \sim 5.5$ and $\lambda_{\mathrm{SO}} \sim$ 1 suggesting that the upper critical field is dominated by Pauli paramagnetic effects ${ }^{41}$.

In stark contrast to the behaviour found in thick devices, the thinnest measured device with $t=14 \mathrm{~nm}$ exhibits a drastically different temperature dependence of the upper critical field for $H_{\mathrm{c} 2} \|(a b)$, reaching a relatively high value of $\sim 12 \mathrm{~T}$ at $2 \mathrm{~K}$ despite the strongly suppressed $T_{\mathrm{c}} \sim 3.63 \mathrm{~K}$. As a result, the slope close to
$T_{c}$ increases dramatically, predicting extremely large orbital limiting field $H_{\mathrm{c} 2}^{\text {orb }}=-0.69\left|\mathrm{~d} H_{\mathrm{c} 2} / \mathrm{d} T\right|_{T=T_{\mathrm{c}}} T_{\mathrm{c}}$ (94 $\mathrm{T}$ as shown in Supplementary Table S1) for $H \|(a b)$ and the Maki parameter becomes extremely large, $a_{\mathrm{M}} \sim 11$. This makes the WHH model less suitable to describe the experimental data of the thinnest flakes. Instead, we use a 2D Ginzburg-Landau (GL) theory ${ }^{42}$, which predicts a square root temperature dependence of the in-plane $H_{c 2}$ close to $T_{c}$. This accurately describes the observed behaviour of the $t=14 \mathrm{~nm}$ device, as shown in Fig. $3 \mathrm{~d}$ by the solid line and in Supplementary Fig. S5. This finding further emphasises the change in character of superconductivity of FeSe in the thin limit, determined by a highly two-dimensional Fermi surface.

\section{DISCUSSION}

In order to compare the effect of thickness on the upper critical field of FeSe devices we investigate a reduced upper critical field phase diagram, by normalising the upper critical field as $h=H_{\mathrm{c} 2} /$ $H_{\mathrm{P}}(0)$ against the reduced temperature $T / T_{\mathrm{C}}$ of each device, as shown in Fig. 4a. Here, the BCS Pauli paramagnetic limit is defined in the weak coupling limit as $H_{\mathrm{p}}(0)=1.85 T_{\mathrm{c}}^{43}$. Interestingly, for thick devices we observe a similar temperature dependence of the reduced upper critical field for each orientation that can be well described by the WHH model. Furthermore, the in-plane upper critical field at zero temperature exceeds the BCS Pauli limit, $H_{\mathrm{c} 2}(0)$ $\sim 1.6 H_{\mathrm{P}}(0)$ for thick flakes and increases above 2 for the thinnest $t=14 \mathrm{~nm}$ flake (Fig. 4a). However, FeSe is an unconventional multi-band superconductor which is expected to deviate from the BCS limit. The Pauli limiting field can exceed the single-band estimate since there are several anisotropic gaps but a single $T_{\mathrm{c}}{ }^{44}$, and therefore one could expect that the largest gap sets the Pauli limit ${ }^{45,46}$. Considering the largest value of the superconducting gap of bulk FeSe of $\Delta \sim 2 \mathrm{meV}^{44}$, the ratio $2 \Delta /\left(k_{\mathrm{B}} T_{\mathrm{c}}\right) \sim 5.3$ is specific to the strong coupling limit. As a result, the Pauli limiting field can reach a value determined by $\sim \Delta \sqrt{g} \mu_{B}$ of $24.4 \mathrm{~T}$ for $g=2^{42}$, which is close to the in-plane upper critical field of bulk FeSe ${ }^{47}$. As the inplane critical field of FeSe thin flakes is reduced to $20 \mathrm{~T}$ or below one can envisage that the dominant gap $\Delta$ is reduced as well, as observed for the thicker flakes (see Fig. 3d). Assuming a fixed coupling constant, we would then expect that the bulk Pauli limiting field of $24.4 \mathrm{~T}$ to be reduced to approximately $10 \mathrm{~T}$ in the $14 \mathrm{~nm}$ flake, lower than our measured values of in-plane $H_{\mathrm{c} 2}$ at low temperature (see Fig. 3d).

Exceeding the BCS Pauli paramagnetic limit coupled with a large value of the Maki parameter (without a finite $\lambda_{\text {so }}$ ) can create the conditions in a clean system with shallow electronic bands for a first-order transition at low temperatures, known as an FFLO state ${ }^{48,49}$. An FFLO state is characterised by a real-space modulation of the superconducting order parameter either in amplitude or phase such that the system energy is minimised under the constraints of a large Zeeman energy and superconducting condensation energy ${ }^{45-48,49}$. Bulk FeSe is a clean system and it has a least one electron-like shallow band and an FFLO sate was recently suggested to occur ${ }^{22,47}$, which manifests as a drastic upturn in the in-plane upper critical field at low temperatures, but is not observed in our thin flake devices. In monolayer systems such as $\mathrm{NbSe}_{2}{ }^{14}$, and ion gated $\mathrm{MoS}_{2}{ }^{50}$, intrinsic spin-orbit interaction effects can lead to Ising superconductivity and a significant increase of $H_{\mathrm{c} 2} \|(a b)$, a factor 6.5 above $H_{\mathrm{P}}(0)^{14}$. As FeSe flakes retain inversion symmetry at all thicknesses due to the lack of interfacial effects, this mechanism cannot explain the enhancement of $H_{\mathrm{c} 2}(a b) / H_{\mathrm{P}}(0)$ in the $t=14 \mathrm{~nm}$ device.

Figure $4 \mathrm{~b}$ summarises the thickness dependence of the superconducting critical temperature $T_{c}$ and the upper critical field anisotropy parameter $\Gamma$ at $T=0.9 T_{\mathrm{c}}$. We observe that while $\Gamma$ increases, the critical temperature $T_{\mathrm{c}}$ decreases suggesting the evolution towards two-dimensional superconductivity in the thin 
limit of FeSe flakes. To understand this further, we use the Ginzburg-Landau formalism to estimate the coherence lengths from the slope of the upper critical value near $T_{\mathrm{c}}$ for the two magnetic field orientations in Fig. $3 d$. Figure $4 c$ shows that the coherence length in the $(a b)$ plane, $\xi_{a b}$, exponentially increases from $\sim 4 \mathrm{~nm}$ to a value of $\sim 10 \mathrm{~nm}$ in the thin limit. These coherence lengths were estimated using the 3D GL theory and are a factor of 2 larger than the values extracted using 2D GL theory in the thin limit (see Fig. 4c). In contrast, $\xi_{c}$ decreases significantly as the flakes get thinner, to $\xi_{c} \sim 0.6 \mathrm{~nm}$ when $t=14 \mathrm{~nm}$, much smaller than the bulk value of $\xi_{c} \sim 1-2 \mathrm{~nm}^{51}$, and is comparable in length to the $c$-axis lattice constant of $\sim 0.55 \mathrm{~nm}$, providing further evidence that the superconductivity is becoming increasingly twodimensional, by confining the order parameter in one unit cell of FeSe. In this case, the weak Josephson coupling of the $(a b)$ planes strongly reduces the role of orbital pair-breaking effects on $H_{\mathrm{c} 2}$. This result is somewhat surprising, as a $t=14 \mathrm{~nm}$ flake is composed of approximately 25 individual FeSe layers, well above the FeSe monolayer limit and not comparable in thickness to the bulk value of $\xi_{c}$. At low temperatures the in-plane upper critical field is significantly suppressed due to Pauli limiting effects and the coherence length, $\xi_{c}$, is likely to increase, as shown in Fig. 11.

The superconductivity in two-dimensional superconductors can also be suppressed by disorder. In the 2D limit, conduction electrons can be easily localised due to the quantum interference effect in the presence of disorder that give rise to Anderson localisation $^{52}$. As the degree of disorder increases, the superconductivity can be destroyed due to the suppression of amplitude of the superconducting order parameter or when the phase fluctuates strongly and its coherence is lost. Despite a reduced $T_{c}$ and lower RRR when compared to bulk, the normal state sheet resistance of the $t=14 \mathrm{~nm}$ device is $\sim 233 \Omega / \square$ and remains well below the quantum resistance $\left(R_{Q}=h / 4 e^{2}=6.45 \mathrm{k} \Omega / \square\right)$ at which a superconductor-insulator transition is expected to occur $^{36}$. This indicates that the suppression of $T_{\mathrm{c}}$ is not driven by disorder, as was previously reported in amorphous thin films ${ }^{33}$. In order to ensure that the observed suppression of superconductivity and broadening effects are not extrinsic, we have examined the effect of air exposure on $T_{c}$, shown in Supplementary Fig. S2. We find that the encapsulated FeSe thick flakes are quite robust to air exposure whereas the thinner ones are more sensitive. However, the timescale required to significantly reduce $T_{c}$ is much longer than that used in our study (which was less than $1 \mathrm{~h}$ ).

Another important parameter that can affect the superconducting and transport behaviour of thin flakes of FeSe is the strain induced by the substrate and its changes with temperature. Recent work on thin films of FeSe showed that positive in-plane strain enhances $T_{\mathrm{c}}$, whilst reducing the structural transition at $T_{\mathrm{s}}{ }^{11}$. This indicates that the suppression of both superconductivity and the structural transition in flakes cannot be solely attributed to inplane strain effects from the substrate. However, the substrate inherently affects the thin flakes and can play a role in determining the local microstructure of the nucleated twin domain structure, and may lead to broader superconducting transitions in thinner samples.

The superconducting anisotropy of almost 10 detected in FeSe thin flakes is large compared with bulk FeSe and flakes of $\mathrm{FeTe}_{0.55} \mathrm{Se}_{0.45}$ of similar thickness. However, large anisotropy is also found in the ultra thin limit of a $1 \mathrm{~nm}$ FeSe EDLT device with a large $T_{\mathrm{c}} \sim 40 \mathrm{~K}^{53}$, suggesting that the character of the twodimensional superconductivity is not changed by gating and doping of charge carriers. Furthermore, the presence of the strong two-dimensional 2D superconducting fluctuations seems to be a signature of multi-band iron-based superconductors and it was found in $500 \mathrm{~nm}$ thick films of FeSe ${ }^{33}$. To reconcile the twodimensional nature of SC fluctuations with the dominant twodimensional behaviour found for the upper critical field of the thinnest flakes, the $2 \mathrm{D}$ fluctuations need to dominante the superconductivity in multiband iron-based superconductors like FeSe (see Fig. 10), and they can originate from a single purely 2D band. Such a 2D band might be derived from in-plane $d_{x y}$ orbitals ${ }^{22}$, but it may be only one of the two bands that dominate the upper critical field of FeSe (Fig. 10). Thus, the multi-band superconductivity of FeSe requires further theoretical understanding to elucidate the evolution of the multi-band models from bulk towards the two-dimensional limit.

The reduced dimensionality of thin flakes together with the short coherence lengths can enhance the thermal fluctuations of the superconducting order parameter near $T_{\mathrm{c}}$ in comparison to classical superconductors ${ }^{54}$. In thin flakes of FeSe the type of fluctuations described by the Ginzburg number (that can be also related to $\left.\left(k_{\mathrm{B}} T_{\mathrm{c}} / E_{\mathrm{F}}\right)^{4}\right)$ can be large due to the small Fermi energy of $\mathrm{FeSe}^{22}$. This number increases upon reduction of the flake thickness, approaching values similar to those found in cuprates ${ }^{54}$. The presence of these fluctuations coupled with the observation of the BKT transition in the thinnest flakes supports the idea that by thinning down $\mathrm{FeSe}$, one stabilises a fluctuating twodimensional and highly anisotropic superconductor. The suppression of superconductivity can be either linked to strong fluctuations or potentially to the loss of Josephson coupling between conducting layers. As flakes become thinner, screening of the Coulomb interaction becomes weaker and eventually the superconductivity is destroyed. For a system with a very small Fermi energy, such as FeSe, this mechanism is expected to be particularly pronounced. It remains to be understood how this type of superconductor interacts with a substrate to drive high- $T_{c}$ superconductivity towards the single atomic layer limit. The interface between the FeSe monolayer and the $\mathrm{SrTiO}_{3}$ substrate also plays an important a role in superconductivity due to the strain caused by the lattice mismatch, enhancement of electronphonon coupling, polaronic effects associated with the high dielectric constant of the substrate, and carrier doping from the interface ${ }^{5,55}$.

In summary, we have investigated the evolution of superconductivity in high quality FeSe thin flakes devices as a function of thickness. We have observed signatures of the BKT transition for both thin and thick flakes suggesting that a highly twodimensional pairing channel dominates superconductivity in FeSe. On the other hand, the upper critical field for thick flakes shows similar behaviour to that of bulk FeSe, and only in the thin limit the upper critical field becomes highly anisotropic. Our studies indicate that in the absence of a dopant substrate, the multi-band superconductivity of FeSe thin flakes has a dominant twodimensional character, but the upper critical field is strongly affected by multiband effects. This supports the premise that enhanced strong two-dimensional fluctuations of FeSe could be one of the key components of this multi-band superconductor possibly stabilised by different orbital effects. Future studies are needed to assess independently the role of strain and carrier doping in stabilising the robust high-temperature superconducting state in FeSe.

\section{METHODS}

Thin FeSe flakes were mechanically exfoliated from high quality single crystals onto silicone elastomer polydimethylsiloxane (PDMS) stamps. Flakes of suitable geometry and thickness were then transferred onto $\mathrm{Si}$ / $\mathrm{SiO}_{2}$ (300 nm oxide) substrates with pre-patterned Au contacts using a dry transfer set-up housed in a nitrogen glovebox with an oxygen and moisture content $<1 \mathrm{ppm}$. To minimise environmental exposure a thin capping layer $(\sim 20 \mathrm{~nm})$ of hexagonal boron nitride (h-BN) was then transferred on top of the FeSe flake, encapsulating the sample. An optical image of a typical sample is shown in the inset of Fig. 1d. The thickness of each sample was accurately determined by atomic force microscopy (AFM) after all measurements had been completed. Error bars in thickness were determined by taking the average of multiple scans across each flake. Typical contact resistances were of the order of 100-1000 Ohms at base 
temperature, depending on the thickness of the FeSe flake, and the size of the contacts. Magneto-transport measurements at temperatures down to $2 \mathrm{~K}$ and magnetic fields up to $16 \mathrm{~T}$ were performed using a Quantum Design Physical Property Measurement System (PPMS), with an additional sample measured at temperatures down to $0.37 \mathrm{~K}$ and magnetic fields up to $37.5 \mathrm{~T}$ at the High Field Magnet Laboratory (HFML-EMFL) in Nijmegen (see Fig. 4). The Hall and longitudinal resistivity contributions were separated by (anti)symmetrizing the data using 4-point measurements obtained under negative and positive magnetic fields. The devices presented are of high quality having a relatively high residual resistance ratio (RRR), $R(300 \mathrm{~K}) / R(15 \mathrm{~K}) \sim 6-16$, as detailed in Supplementary Fig. S3. The superconducting critical temperature, $T_{c}$, and upper critical field, $H_{\mathrm{c} 2}$ was normally defined as the position at which the resistance reached $50 \%$ of its normal state value or the maximum in its derivative. Error bars in Fig. $1 \mathrm{~d}$ and Fig. $4 \mathrm{~b}$ are defined by the onset and offset values of the superconducting critical temperature as shown in Fig. 8. The upper critical field was measured for two different orientations of the conducting $(a b)$ plane with respect to the applied magnetic field (either parallel to the conducting plane, $H \|(a b)\left(\theta=90^{\circ}\right)$ or perpendicular to it, and parallel to the crystallographic $c$-axis, $H \| c\left(\theta=0^{\circ}\right)$ ). Angular-dependent studies were also performed at $2 \mathrm{~K}$. Error bars in Fig. $3 \mathrm{c}$ and Fig. $4 \mathrm{c}$ are given by uncertainty in linear fits to the field induced resistive transition.

\section{DATA AVAILABILITY}

The data that support the findings of this study are available from the corresponding author upon reasonable request. In accordance with the EPSRC policy framework on research data, access to the raw data will be made available through ORA depository at the University of Oxford at https://doi.org/10.5287/bodleian:o0q7YPNzm.

Received: 30 July 2019; Accepted: 30 March 2020;

Published online: 15 May 2020

\section{REFERENCES}

1. Hsu, F.-C. et al. Superconductivity in the PbO-type structure FeSe. Proc. Natl Acad. Sci. USA 105, 14262-14264 (2008).

2. Wang, Q. Y. et al. Interface-induced high-temperature superconductivity in single unit-cell FeSe films on $\mathrm{SrTiO}_{3}$. Chin. Phys. Lett. 29, 37402 (2012).

3. $\mathrm{He}, \mathrm{S}$. et al. Phase diagram and electronic indication of high-temperature superconductivity at $65 \mathrm{~K}$ in single-layer FeSe films. Nat. Mater. 12, 605-610 (2013).

4. Ge, J. F. et al. Superconductivity above $100 \mathrm{~K}$ in single-layer FeSe films on doped $\mathrm{SrTiO}_{3}$. Nat. Mater. 14, 285-289 (2015).

5. Huang, D. \& Hoffman, J. E. Monolayer FeSe on $\mathrm{SrTiO}_{3}$. Annu. Rev. Condens. Matter Phys. 8, 311-336 (2017).

6. Geim, A. K. \& Grigorieva, I. V. Van der Waals heterostructures. Nature 499, 419-425 (2013).

7. Novoselov, K. S., Mishchenko, A., Carvalho, A. \& CastroNeto, A. H. 2D materials and van der Waals heterostructures. Science 353, aac9439 (2016).

8. Tan, S. et al. Interface-induced superconductivity and strain-dependent spin density waves in $\mathrm{FeSe} / \mathrm{SrTiO}_{3}$ thin films. Nat. Mater. 12, 634-640 (2013).

9. Wang, Q. et al. Thickness dependence of superconductivity and superconductorinsulator transition in ultrathin FeSe films on $\mathrm{SrTiO}_{3}(001)$ substrate. $2 \mathrm{D}$ Mater. 2, 044012 (2015)

10. Sun, Y. et al. High temperature superconducting FeSe films on $\mathrm{SrTiO}_{3}$ substrates. Sci. Rep. 4, 6040 (2014).

11. Nabeshima, F., Imai, Y., Hanawa, M., Tsukada, I. \& Maeda, A. Enhancement of the superconducting transition temperature in FeSe epitaxial thin films by anisotropic compression. Appl. Phys. Lett. 103, 172602 (2013)

12. Schneider, R., Zaitsev, A. G., Fuchs, D. \& Löhneysen, H. V. Superconductorinsulator quantum phase transition in disordered FeSe thin films. Phys. Rev. Lett. 108, 257003 (2012).

13. Feng, Z. et al. Tunable critical temperature for superconductivity in FeSe thin films by pulsed laser deposition. Sci. Rep. 8, 4039 (2018).

14. Xi, X. et al. Ising pairing in superconducting $\mathrm{NbSe}_{2}$ atomic layers. Nat. Phys. 12, 139-143 (2016).

15. Navarro-Moratalla, E. et al. Enhanced superconductivity in atomically thin $\mathrm{TaS}_{2}$. Nat. Commun. 7, 11043 (2016).

16. Jiang, $\mathrm{D}$. et al. High- $T_{c}$ superconductivity in ultrathin $\mathrm{Bi}_{2} \mathrm{Sr}_{2} \mathrm{CaCu}_{2} \mathrm{O}_{8}$. $x$ down to half-unit-cell thickness by protection with graphene. Nat. Commun. 5, 5708 (2014).
17. Lei, B. et al. Evolution of high-temperature superconductivity from a low- $T_{c}$ phase tuned by carrier concentration in FeSe thin flakes. Phys. Rev. Lett. 116, 077002 (2016).

18. Lei, B. et al. Tuning phase transitions in FeSe thin flakes by field-effect transistor with solid ion conductor as the gate dielectric. Phys. Rev. B 95, 020503 (2017).

19. Ying, T. P. et al. Discrete superconducting phases in FeSe-derived superconductors. Phys. Rev. Lett. 121, 207003 (2018).

20. Yang, R., Luo, W., Chi, S., Bonn, D. \& Xia, G. The stability of exfoliated FeSe nanosheets during in-air device fabrication processes. IEEE T Nanotechnol. 18, 37-41 (2018).

21. Castellanos-Gomez, A. et al. Deterministic transfer of two-dimensional materials by all-dry viscoelastic stamping. 2D Mater. 1, 011002 (2014).

22. Coldea, A. I. \& Watson, M. D. The key ingredients of the electronic structure of FeSe. Annu. Rev. Cond. Matt. Phys. 9, 125-146 (2018).

23. Schoop, L. M. et al. Pressure-restored superconductivity in Cu-substituted FeSe. Phys. Rev. B 84, 174505 (2019).

24. Watson, M. D. et al. Dichotomy between the hole and electron behavior in multiband superconductor fese probed by ultrahigh magnetic fields. Phys. Rev. Lett. 115, 027006 (2015).

25. Cooper, L. N. Superconductivity in the neighborhood of metallic contacts. Phys. Rev. Lett. 6, 689-690 (1961).

26. Simonin, J. Surface term in the superconductive ginzburg-landau free energy: application to thin films. Phys. Rev. B 33, 7830-7832 (1986).

27. Lin, Z. et al. Quasi-two-dimensional superconductivity in $\mathrm{FeSe}_{0} .3 \mathrm{Te}_{0} .7$ thin films and electric-field modulation of superconducting transition. Sci. Rep. 5, 14133 (2015).

28. Berezinskii, V. L. Destruction of long-range order in one-dimensional and twodimensional systems possessing a continuous symmetry group. II. Quantum Syst. J. Exp. Theor. Phys. 34, 1144-1156 (1971).

29. Kosterlitz, D. J. \& Thouless, J. M. Ordering, metastability and phase transitions in two-dimensional systems. J. Phys. C. Solid State Phys. 1181, 1181-1203 (1973).

30. Hebard, A. F. \& Fiory, A. T. Critical-exponent measurements of a two-dimensional superconductor. Phys. Rev. Lett. 50, 1603-1606 (1983).

31. Garland, J. C. \& Lee, H. J. Influence of a magnetic field on the two-dimensional phase transition in thin-film superconductors. Phys. Rev. B 36, 3638-3650 (1987).

32. Zhang, W. H. et al. Direct observation of high-temperature superconductivity in one-unit-cell FeSe films. Chin. Phys. Lett. 31, 017401 (2014).

33. Schneider, R., Zaitsev, A. G., Fuchs, D. \& VonLöhneysen, H. Excess conductivity and Berezinskii-Kosterlitz-Thouless transition in superconducting FeSe thin films. J. Phys. Condens. Matter 26, 455701 (2014).

34. Rullier-Albenque, F., Colson, D., Forget, A. \& Alloul, H. Multiorbital effects on the transport and the superconducting fluctuations in LiFeAs. Phys. Rev. Lett. 109, 187005 (2012).

35. Venditti, G. et al. Non-linear IV characteristics in two-dimensional superconductors: Berezinskii-Kosterlitz-Thouless physics vs inhomogeneity. Phys. Rev. B 100, 064506 (2019).

36. Markovic, N., C.Christiansen, A. M., Mack, W. H. \& Huber, A. M. G. Superconductorinsulator transition in two dimensions. Phys. Status Solidi Basic Res. 60, 4320-4328 (1999).

37. Bennemann, K.H. \& Ketterson, J.B. Superconductivity (Springer, 2008).

38. Audouard, A. et al. Quantum oscillations and upper critical magnetic field of the iron-based superconductor FeSe. EPL 109, 27003 (2015).

39. Borg, C. K. et al. Strong anisotropy in nearly ideal tetrahedral superconducting FeS single crystals. Phys. Rev. B 93, 094522 (2016).

40. Werthamer, N. R., Helfand, E. \& Hohenberg, P. C. Temperature and purity dependence of the superconducting critical field, $H_{c} 2$. III. Electron spin and spinorbit effects. Phys. Rev. 147, 295-302 (1966).

41. Khim, S. et al. Evidence for dominant Pauli paramagnetic effect in the upper critical field of single-crystalline $\mathrm{FeSe}_{0} .6 \mathrm{Se}_{0}$.4. Phys. Rev. B 81, 184511 (2010).

42. Tinkham, M. Introduction to Superconductivity (Dover Publications, 1996).

43. Clogston, A. M. Upper limit for the critical field in hard superconductors. Phys. Rev. Lett. 9, 266-267 (1962).

44. Sprau, P. O. et al. Discovery of orbital-selective cooper pairing in FeSe. Science 357, 75-80 (2017)

45. Gurevich, A. Upper critical field and the Fulde-Ferrel-Larkin-Ovchinnikov transition in multiband superconductors. Phys. Rev. B 82, 184504 (2010).

46. Gurevich, A. Challenges and opportunities for applications of unconventional superconductors. Annu. Rev. Cond. Matt. Phys. 5, 35-56 (2014).

47. Kasahara, S. et al. Evidence for an FFLO state with segmented vortices in the BCSBEC-crossover superconductor FeSe. Phys. Rev. Lett. 124, 107001 (2020).

48. Fulde, P. \& Ferrell, R. A. Superconductivity in a strong spin-exchange field. Phys. Rev. 135, A550-A563 (1964).

49. Larkin, A. I. \& Ovchinnikov, Y. N. Nonuniform state of superconductors. Zh. Eksp. Teor. Fiz. 47, 1136-1146 (1964).

50. Lu, J. M. et al. Evidence for two-dimensional Ising superconductivity in gated $\mathrm{MoS}_{2}$. Science 350, 1353-1357 (2015). 
51. Terashima, T. et al. Anomalous Fermi surface in FeSe seen by Shubnikov-de Haas oscillation measurements. Phys. Rev. B 90, 144517 (2014).

52. Anderson, P. W. Absence of diffusion in certain random lattices. Phys. Rev. 109, 1492-1505 (1958).

53. Shiogai, J., Kimura, S., Awaji, S., Nojima, T. \& Tsukazaki, A. Anisotropy of the upper critical field and its thickness dependence in superconducting FeSe electricdouble-layer transistors. Phys. Rev. B 97, 174520 (2018).

54. Blatter, G., Feigel'man, M. V., Geshkenbein, V. B., Larkin, A. I. \& Vinokur, V. M. Vortices in high-temperature superconductors. Rev. Mod. Phys. 66, 1125-1388 (1994).

55. Wang, Z., Liu, C., Liu, Y. \& Wang, J. High-temperature superconductivity in oneunit-cell FeSe films. J. Phys. 29, 153001 (2017).

\section{ACKNOWLEDGEMENTS}

We thank Lara Benfatto for very helpful comments on our manuscript and Sid Parameswaran, Steve Simon for useful discussions. The research was funded by the Oxford Centre for Applied Superconductivity (CFAS) at Oxford University. We also acknowledge financial support of the John Fell Fund of the Oxford University. This work was partly supported by EPSRC (EP/I004475/1, EP/I017836/1). L.F. is supported by the Bath/Bristol Centre for Doctoral Training in Condensed Matter Physics, under the EPSRC (UK) Grant No. EP/L015544. Part of this work was supported by HFML-RU/ FOM and LNCMI-CNRS, members of the European Magnetic Field Laboratory (EMFL) and by EPSRC (UK) via its membership to the EMFL (EP/N01085X/1). A.A.H acknowledges the financial support of the Oxford Quantum Materials Platform Grant (EP/M020517/1). A.I.C. acknowledges an EPSRC Career Acceleration Fellowship (EP/I004475/1)

\section{AUTHOR CONTRIBUTIONS}

L.F. has prepared the thin flake devices. L.F. and M.B. have performed experiments in Oxford. A.I.C., M.B. and A.M. performed experiments in Nijmegen. A.A.H. grew single crystals. L.F., S.J.B. and M.B. performed the data analysis. L.F., S.J.B. and A.I.C. wrote the paper with contributions and comments from all the authors.

\section{COMPETING INTERESTS}

The authors declare no competing interests.

\section{ADDITIONAL INFORMATION}

Supplementary information is available for this paper at https://doi.org/10.1038/ s41535-020-0227-3.

Correspondence and requests for materials should be addressed to L.S.F. or A.I.C.

Reprints and permission information is available at http://www.nature.com/ reprints

Publisher's note Springer Nature remains neutral with regard to jurisdictional claims in published maps and institutional affiliations.

(i) Open Access This article is licensed under a Creative Commons Attribution 4.0 International License, which permits use, sharing, adaptation, distribution and reproduction in any medium or format, as long as you give appropriate credit to the original author(s) and the source, provide a link to the Creative Commons license, and indicate if changes were made. The images or other third party material in this article are included in the article's Creative Commons license, unless indicated otherwise in a credit line to the material. If material is not included in the article's Creative Commons license and your intended use is not permitted by statutory regulation or exceeds the permitted use, you will need to obtain permission directly from the copyright holder. To view a copy of this license, visit http://creativecommons. org/licenses/by/4.0/.

(c) The Author(s) 2020 